\title{
Tau Antibody Targeting Pathological Species Blocks Neuronal Uptake and Interneuron Propagation of Tau in Vitro
}

Chloe K. Nobuhara, ${ }^{*}$ Sarah L. DeVos, ${ }^{*}$ Caitlin Commins, ${ }^{*}$ Susanne Wegmann, ${ }^{*}$ Benjamin D. Moore, * Allyson D. Roe, * Isabel Costantino, ${ }^{*}$ Matthew P. Frosch, ${ }^{*}$ Rose Pitstick, ${ }^{\dagger}$ George A. Carlson, ${ }^{\dagger}$ Christoph Hock, ${ }^{\ddagger \delta}$ Roger M. Nitsch, ${ }^{\ddagger \delta}$ Fabio Montrasio, ${ }^{\ddagger}$ Jan Grimm, ${ }^{\ddagger}$ Anne E. Cheung, " Anthone W. Dunah, " Marion Wittmann, "T Thierry Bussiere, " Paul H. Weinreb, " Bradley T. Hyman, ${ }^{*}$ and Shuko Takeda*

From the Alzheimer's Disease Research Laboratory, * Department of Neurology, MassGeneral Institute for Neurodegenerative Disease, Massachusetts General Hospital, Harvard Medical School, Charlestown, Massachusetts; the McLaughlin Research Institute, ${ }^{\dagger}$ Great Falls, Montana; Neurimmune, ${ }^{\ddagger}$ Schlieren, Switzerland; the Institute for Regenerative Medicine, ${ }^{\S}$ University of Zurich, Zurich, Switzerland; and Biogen Inc., "Cambridge, Massachusetts

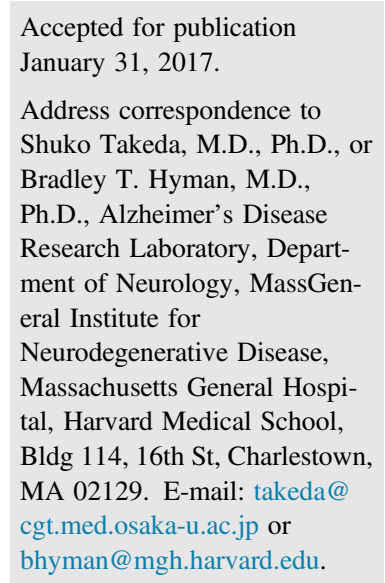

\begin{abstract}
The clinical progression of Alzheimer disease (AD) is associated with the accumulation of tau neurofibrillary tangles, which may spread throughout the cortex by interneuronal tau transfer. If so, targeting extracellular tau species may slow the spreading of tau pathology and possibly cognitive decline. To identify suitable target epitopes, we tested the effects of a panel of tau antibodies on neuronal uptake and aggregation in vitro. Immunodepletion was performed on brain extract from tau-transgenic mice and postmortem $A D$ brain and added to a sensitive fluorescence resonance energy transfer-based tau uptake assay to assess blocking efficacy. The antibodies reduced tau uptake in an epitope-dependent manner: N-terminal (Tau13) and middomain (6C5 and HT7) antibodies successfully prevented uptake of tau species, whereas the distal C-terminal-specific antibody (Tau46) had little effect. Phosphorylation-dependent (40E8 and p396) and C-terminal half (4E4) tau antibodies also reduced tau uptake despite removing less total tau by immunodepletion, suggesting specific interactions with species involved in uptake. Among the seven antibodies evaluated, 6C5 most efficiently blocked uptake and subsequent aggregation. More important, $6 \mathrm{C} 5$ also blocked neuron-to-neuron spreading of tau in a unique three-chamber microfluidic device. Furthermore, $6 \mathrm{C} 5$ slowed down the progression of tau aggregation even after uptake had begun. Our results imply that not all antibodies/epitopes are equally robust in terms of blocking tau uptake of human AD-derived tau species. (Am J Pathol 2017, 187: 1399-1412; http://dx.doi.org/10.1016/j.ajpath.2017.01.022)
\end{abstract}

The intracellular aggregation of microtubule-associated protein tau as neurofibrillary tangles (NFTs) is a hallmark of Alzheimer disease (AD) pathology. ${ }^{1,2}$ The clinical progression of $\mathrm{AD}$ is most tightly linked with the accumulation of NFTs, which start in the entorhinal cortex and spread throughout the brain in a stereotyped manner. ${ }^{3}$ Recent studies suggest that interneuronal transfer of pathological tau species underlies the spreading of NFTs in the brain of

\footnotetext{
Supported by NIH grant AG026249 (B.T.H.), Massachusetts Alzheimer's Disease Research Center grant P50AG05134 (B.T.H.), the Massachusetts Life Sciences Center (B.T.H.), the Japan Society for the Promotion of Science fellowship (S.T.), NIH T32 training grant Training in the Molecular Biology of Neurodegeneration (S.L.D.), and the German Research Foundation fellowship (S.W.).

Disclosures: Supported in part by a research agreement to Massachusetts General Hospital from Biogen, Inc. A.E.C., A.W.D., and T.B. are current employees and shareholders of Biogen; M.W. is a former employee and
}

shareholder of Biogen and currently an employee and shareholder of Praxis Precision Medicines; F.M. is a current employee and shareholder of Neurimmune; J.G. is an officer, board member, and shareholder of Neurimmune; C.H. is a former board member and shareholder of Neurimmune; R.M.N. is president and shareholder of Neurimmune.

Current address of S.T., Department of Clinical Gene Therapy, Center of Medical Innovation and Translational Research, School of Medicine, Osaka University, Suita, Japan. 
AD patients. ${ }^{4-7}$ These findings support the idea that clearing the soluble extracellular tau species responsible for propagation may affect the clinical progression of $\mathrm{AD}$.

Immunotherapies targeting tau have been extensively studied both in vitro and in vivo, and encouraging results have been reported on the antibody-mediated removal of the pathological form of tau. ${ }^{8}$ Various tau antibodies directed against distinct epitopes have been tested, including N-terminal antibodies, ${ }^{9,10}$ phospho-tau-specific antibodies, ${ }^{11-15}$ and conformation-dependent antibodies. ${ }^{12,16}$ Some of these antibodies appear to ameliorate the cognitive impairments as well as the neuropathology of tau-transgenic mice; however, it is still controversial which antibody/ epitope can most efficiently and selectively remove tau species involved in $\mathrm{AD}$ pathogenesis. Both intracellular and extracellular tau proteins are known to carry multiple posttranslational modifications, such as truncation and phosphorylation, ${ }^{17-20}$ which are thought to contribute to $\mathrm{AD}$ pathogenesis. The $\mathrm{AD}$ brain likely contains a heterogeneous pool of pathological forms of tau; thus, identification of appropriate epitopes in a specific tau species is essential to optimizing a tau immunotherapy strategy. In addition, the effect of tau antibodies on the propagation phenomenon remains largely unclear.

In this study, to identify suitable target epitopes and antibodies against the tau species involved in uptake, we tested the effects of a panel of tau antibodies on neuronal tau uptake in vitro: phosphorylation-independent antibodies against N-terminal (Tau13); middomain (6C5 and HT7), C-terminal (4E4), and C-terminal end (Tau46) epitopes; phosphorylation-dependent antibodies against phosphoserine 202 and phospho-threonine 205 (40E8); and phospho-serine 396 (p396). We used a sensitive fluorescence resonance energy transfer (FRET)-based assay to detect tau uptake and subsequent intracellular aggregation in mouse primary neuron culture. Phosphate-buffered saline (PBS)-soluble fraction of brain extracts from tau-transgenic mice $\left(\mathrm{rTg} 4510^{21}\right)$ and human $\mathrm{AD}$ postmortem cortices were used; a bioactivity assay in cultured primary neurons was used to measure tau uptake and aggregation. For the human $\mathrm{AD}$ brain experiment, we used high-molecular-weight (HMW) tau previously identified as a relevant bioactive species that can be readily taken up by neurons and synaptically propagated to other neurons. ${ }^{4}$ Furthermore, we examined the effect of tau antibody (6C5) on the transfer of tau between neurons using a unique microfluidic neuron chamber. ${ }^{4}$ Our results suggest that not all tau epitopes are equally robust in terms of blocking neuronal uptake of $\mathrm{AD}$ brain-derived tau.

\section{Materials and Methods}

\section{Animals}

Eleven- to thirteen-month-old $\mathrm{rTg} 4510$ and control animals were used. The $\mathrm{rTg} 4510$ mouse overexpresses full-length human four-repeat tau (0N4R) with the P301L frontotemporal dementia mutation. ${ }^{21}$ Both male and female mice were used. All experiments were performed under national (US NIH) and institutional (Massachusetts General Hospital Subcommittee for Research Animal Care and the Institutional Animal Care and Use Committee at Harvard Medical School) guidelines. All animal experiments were approved by the Massachusetts General Hospital and McLaughlin Research Institute Institutional Animal Care and Use Committees.

\section{Human Brain Samples}

Frozen frontal cortex tissues of four AD patients were obtained from the Massachusetts Alzheimer's Disease Research Center Brain Bank. The demographic characteristics of the subjects are shown in Table 1. The Massachusetts General Hospital Institutional Review Board approved the study protocol. All of the AD subjects fulfilled the National Institute on Aging-Reagan criteria for high likelihood of AD. Cortical gray matter was weighed and processed as described in the following section.

\section{Tau Antibodies}

Human monoclonal antibodies 6C5, 40E8, and 4E4 were derived from a deidentified blood lymphocyte library collected from healthy elderly subjects with no signs of cognitive impairment. Supernatants from memory B cells isolated from peripheral blood lymphocyte preparations were screened for the ability to bind recombinant tau and human Alzheimer disease-derived paired helical filament tau. The variable domain regions of selected tau-reactive Bcell clones were sequenced and subcloned into expression constructs engineered to produce recombinant human $\mathrm{IgG1}$ antibodies (P.H.W., F.M., J.G., and T.B., unpublished data). The following commercial antibodies were used: Tau13 (mouse; sc-21796; Santa Cruz Biotechnology, Dallas, TX), Tau46 (mouse; sc-32274; Santa Cruz Biotechnology), HT7 (mouse; MN1000; Thermo Fisher Scientific, Waltham, MA), and pS396 (rabbit; 44752; Life Technologies, Carlsbad, CA). The epitopes recognized and other characteristics of each antibody are shown in Table 2.

Table 1 Characteristics of the Subjects with AD Used in the Study

\begin{tabular}{|c|c|c|c|c|c|}
\hline $\begin{array}{l}\text { Case } \\
\text { no. }\end{array}$ & $\begin{array}{l}\text { Age at } \\
\text { death, } \\
\text { years }\end{array}$ & Sex & $\begin{array}{l}\text { Postmortem } \\
\text { interval, } \\
\text { hours }\end{array}$ & Diagnosis & $\begin{array}{l}\text { Braak } \\
\text { stage, }^{2} \\
\text { CERAD } \\
\text { score }^{22}\end{array}$ \\
\hline 1170 & 80 & Female & 20 & $A D$ & VI, C \\
\hline 1199 & 92 & Female & 12 & $A D$ & VI, C \\
\hline 1222 & 90 & Female & 5 & $A D$ & VI, C \\
\hline 1266 & 51 & Female & 10 & $A D$ & VI, C \\
\hline
\end{tabular}

$A D$, Alzheimer disease; CERAD, Consortium to Establish a Registry for Alzheimer's Disease. 


\section{Tau Binding ELISA}

The apparent affinity of each tau antibody was determined by enzyme-linked immunosorbent assay (ELISA). Plates (Immulon 3855) were coated with $1 \mu \mathrm{g} / \mathrm{mL}$ of recombinant Tau (rPeptide) or $5 \mu \mathrm{g} / \mathrm{mL}$ of paired helical filamentinsoluble tau isolated from human $\mathrm{AD}$ brain homogenate, according to published protocols, ${ }^{23}$ diluted into $100 \mathrm{mmol} / \mathrm{L}$ $\mathrm{NaHCO}_{3}$ (pH 9.6) and incubated overnight at $4{ }^{\circ} \mathrm{C}$. Contents were discarded, and plates were blocked in blocking buffer $(1 \times$ casein diluted $1: 10$ in PBS containing $0.02 \%$ Tween20) for 1 hour at $25^{\circ} \mathrm{C}$. Plates were washed with $0.05 \%$ Tween-20 in PBS, anti-tau antibody diluted in blocking buffer was added at the indicated concentrations, and incubated for 1 hour at $25^{\circ} \mathrm{C}$. After washing, a 1:10,000 dilution in blocking buffer of a horseradish peroxidase-conjugated anti-murine $\operatorname{IgG}$ or anti-rabbit IgG (Jackson ImmunoResearch, West Grove, PA) antibody was incubated on the plate for 30 minutes at room temperature. Plates were washed and developed using 3,3',5,5;-tetramethylbenzidine (BioFX, Owings Mills, MD) for 2 to $5 \mathrm{mi}-$ nutes. The reaction was stopped in $1 \mathrm{~N} \mathrm{H}_{2} \mathrm{SO}_{4}$, and colorometric analysis was performed using a Beckman spectrophotometer at $450 \mathrm{~nm}$.

\section{Primary Cortical Neuron Culture}

Primary cortical neurons were prepared from cerebral cortices of embryonic day (E) 14 to 15 CD1 mouse embryos (Charles River Laboratories, Cambridge, MA), as described previously. ${ }^{4}$ Cortices were dissected and dissociated with a Papain Dissociation System (Worthington Biochemical Corp., Lakewood, NJ), according to the manufacturer's protocol. The dissociated neurons were plated at a density of $6.8 \times 10^{4}$ cells on 96-well poly-D-lysine-coated plates (3603; Corning Inc., Corning, NY) or microfluidic devices (In Vitro Tau Propagation Assay). Cultures were maintained at $37^{\circ} \mathrm{C}$ with $5 \% \mathrm{CO}_{2}$ in Neuro-basal (Life Technologies, Inc.) medium with 2\% (v/v) B27 supplements (Gibco, Grand Island, $\mathrm{NY}$ ), $2 \mathrm{mmol} / \mathrm{L}$ glutamax (Gibco), $100 \mathrm{U} / \mathrm{mL}$ penicillin (Gibco), and $100 \mathrm{~g} / \mathrm{mL}$ streptomycin (Gibco).

\section{Lentivirus Production}

Lentiviruses encoding tau repeat domain (RD) P301L-CFP and tau RD P301L-YFP were generated as described previously, ${ }^{24}$ with minor modifications. Briefly, HEK293T cells were cotransfected with $1200 \mathrm{ng}$ PSP, $400 \mathrm{ng}$ vesicular stomatitis virus-G, and $400 \mathrm{ng}$ FCIV plasmids using TransIT293 Transfection Reagent (MIR2704; Mirus Bio, Madison, WI). Conditioned media were collected 48 hours after transfection and centrifuged at $1000 \times g$ for 5 minutes. Viral supernatants were concentrated using Lenti-X-Concentrator (631231; Clontech, Mountain View, CA), then centrifuged at $3000 \times g$ for 15 minutes. The final pellets were dissolved in PBS and stored at $-80^{\circ} \mathrm{C}$ before use. Lentiviral titers were determined using a p24 ELISA (QuickTiter Lentivirus Titer Kit, VPK-107; Cell Biolabs, San Diego, CA), according to the manufacturer's instruction.

\section{Tau Uptake Assay in Mouse Primary Neurons}

A tau uptake assay was performed as described previously, ${ }^{24}$ with some modifications. Mouse primary neurons were cotransduced with 39 to $42 \mathrm{ng} / \mathrm{mL}$ lentivirus encoding tau RD P301L-CFP and tau RD P301L-YFP on days in vitro (DIV) 1 and treated with $\operatorname{rTg} 4510$ brain extract or HMW size-exclusion chromatography (SEC) fraction of human AD brain extract, with or without immunodepletion, on DIV 6. The rTg4510 brain extract was diluted with culture medium to make a final concentration of $100 \mu \mathrm{g} / \mathrm{mL}$ total protein, and $50 \mu \mathrm{L}$ was added to each well (5 $\mu \mathrm{g}$ total protein/well), unless stated otherwise. HMW SEC fraction of AD brain extract was diluted at 1:2 with culture medium and $50 \mu \mathrm{L}$ was added to each well. For the comparison between the PBS-10,000 $\times g$ extract and the HMW fraction of human $\mathrm{AD}$ brain, each sample was diluted with culture medium to make a final concentration of $100 \mathrm{ng} / \mathrm{mL}$ human tau (measured by human tau ELISA), and $50 \mu \mathrm{L}$ was added to each well. Each sample was filtered through a $0.2-\mu \mathrm{m}$ membrane filter before incubation. Tau seeds were not incubated with protein carriers (eg, lipofectamine) to measure uptake plus seeding. By contrast, incubating tau seeds

Table 2 Tau Antibodies Used in This Study

\begin{tabular}{|c|c|c|c|c|c|}
\hline \multirow[b]{2}{*}{ Antibody } & \multirow[b]{2}{*}{ Isotype } & \multicolumn{2}{|l|}{$\mathrm{EC}_{50}, \mathrm{nmol} / \mathrm{L}$} & \multirow[b]{2}{*}{ Epitope } & \multirow[b]{2}{*}{ Source } \\
\hline & & $\begin{array}{l}\text { Recombinant } \\
\text { tau (ELISA) }\end{array}$ & $\begin{array}{l}\text { PHF tau } \\
\text { (ELISA) }\end{array}$ & & \\
\hline 40E8 & Human $\operatorname{IgG} 1 / \lambda$ & $>500$ & 0.2 & $\begin{array}{l}195-212 \\
(p S 202 \text { and pT205) }\end{array}$ & Biogen \\
\hline 4E4 & Human $\operatorname{IgG} 1 / \lambda$ & 13 & $>100$ & $385-395$ & Biogen \\
\hline Tau46 & Mouse IgG1 & 0.06 & 0.03 & $404-441$ & Santa Cruz Biotechnology \\
\hline pS396 & Rabbit polyclonal & $>500$ & 1.8 & pS396 & Life Technologies (Carlsbad, CA) \\
\hline
\end{tabular}

$\mathrm{EC}_{50}, 50 \%$ effective concentration; ELISA, enzyme-linked immunosorbent assay; PHF, paired helical filament. 
with protein transduction reagents leads to a measure of seeding alone. At the designated time points, confocal images were obtained via a FRET channel (excited with a 458-nm laser, and fluorescence was captured with 500 to $550 \mathrm{~nm}$ filter) using a confocal microscope (Zeiss Axiovert 200 inverted microscope; Carl Zeiss, Peabody, MA). The number of intracellular tau-aggregate positive neurons per well was counted and used for the quantification analysis. Each condition was performed at least in duplicate.

\section{Brain Extraction}

Mice were perfused with cold PBS, and the brain was rapidly excised and frozen in liquid nitrogen, then stored at $-80^{\circ} \mathrm{C}$ until use. Brain tissue was homogenized in 5 volumes (w/v) of cold PBS containing protease inhibitor (5871S; Cell Signaling, Danvers, MA) using a Teflon-glass homogenizer. The homogenate was briefly sonicated (Fisher Scientific Sonic Dismembrator model 100; output 2, $6 \times 1$ second) and centrifuged at $10,000 \times g$ for 10 minutes at $4^{\circ} \mathrm{C}$ (PBS-10,000 $\times g$ extract). The supernatants were collected and stored at $-80^{\circ} \mathrm{C}$ before use.

\section{Size-Exclusion Chromatography}

PBS-soluble $(10,000 \times g)$ extracts from AD postmortem brain tissues were separated by SEC on single Superdex 200 10/300 GL columns (17-5175-01; GE Healthcare, Boston, MA) in PBS (P3813; Sigma-Aldrich, St. Louis, MO; filtered through a $0.2-\mu \mathrm{m}$ membrane filter), at a flow rate of $0.5 \mathrm{~mL} /$ minute, with an AKTA purifier 10 (GE Healthcare). Fractions were collected every 2 minutes $(1 \mathrm{~mL} /$ fraction). Each brain extract was filtered through a $0.2-\mu \mathrm{m}$ membrane filter and $750 \mu \mathrm{L}$ of the filtered sample was loaded onto an SEC column. The individual fractions separated by SEC were analyzed by ELISA [Tau (total) Human ELISA kit]. A small peak of the HMW fraction tau that was eluted in a volume fraction of between 8.0 and $9.0 \mathrm{~mL}$ was used to optimize readouts on the tau uptake assay.

\section{Immunostaining of Brain Sections}

Formalin-fixed, paraffin-embedded human brain tissue from frontal cortex was cut into sections ( $8 \mu \mathrm{m}$ thick) and mounted onto slides. Sections were deparaffinized in xylene for 10 minutes twice at room temperature and a descending ethanol series ( $100 \%$ for 5 minutes $\times 2,95 \%$ for 5 minutes, $70 \%$ for 5 minutes). After briefly rinsing in tris-buffered saline (TBS), sections were blocked in 5\% (w/v) normal goat serum/TBS for 1 hour at room temperature. Previously unpublished human monoclonal primary antibodies 6C5 [total tau (amino acids 125 to 131); 1:500; Biogen, Cambridge, MA) and 40E8 (pS202-pT205 tau; 1:500; Biogen), 4E4 [total tau (amino acids 385 to 395); 1:500; Biogen], or a tau polyclonal antibody (A0024; 1:500; Dako, Glostrup, Denmark) in 2\% normal goat serum/TBS were incubated with sections overnight at $4^{\circ} \mathrm{C}$. Sections were washed three times in TBS for 5 minutes and incubated with horseradish peroxidase-conjugated rabbit anti-human (AB6759; Abcam, Cambridge, MA) secondary antibody (1:200 dilution in 2\% normal goat serum/TBS) for 1 hour at room temperature. After washing in TBS (5 minutes $\times 3$ ), sections were dehydrated and coverslips were mounted using mounting medium.

\section{Immunodepletion}

Immunodepletion of tau from brain extracts was performed using Dynabeads Protein G Immunoprecipitation Kit (10007D; Life Technologies), according to the manufacturer's instructions with minor modifications. Dynabeads Protein G $(0.75 \mathrm{mg})$ was incubated with $2 \mu \mathrm{g}$ of each tau antibody or control IgG for 10 minutes with rotation at room temperature. After washing with $200 \mu \mathrm{L}$ of washing buffer, the Dynabeads-antibody complex was incubated with $200 \mu \mathrm{L}$ of $\operatorname{rTg} 4510$ brain extracts (PBS-10,000 $\times g, 100 \mu \mathrm{g} / \mathrm{mL}$ total protein) or $110 \mu \mathrm{L}$ of $\mathrm{HMW}$ fraction of $\mathrm{AD}$ brain extracts $(\mathrm{PBS}-10,000 \times g$ ) for 10 minutes with rotation at room temperature. Dynabeads-antibody-antigen complex was isolated using a magnetic holder, and the supernatant was collected for tau uptake assay and tau ELISA measurement; human control IgG served as a negative control. In preliminary experiments, we had compared mouse/rabbit control IgG with human control IgG in the same immunodepletion and tau uptake assay protocol. Because no differences in the results of the three control antibodies were observed (data not shown), we used the human control IgG as the negative control for all experiments.

\section{SDS-PAGE and Western Blot}

Protein/lane $(10 \mu \mathrm{g})$ of immunodepleted and original brain homogenate were electrophoresed on $10 \%$ to $20 \%$ Novex Tris-Glycine gels (Life Technologies, Grand Island, NY) in tris-glycine SDS running buffer for SDS-PAGE (Life Technologies). Gels were transferred to polyvinylidene difluoride membranes, and membranes were blocked for 60 minutes at room temperature in $5 \%(\mathrm{w} / \mathrm{v})$ bovine serum albumin $(\mathrm{BSA}) /$ TBS-Tween (TBS-T). Membranes were probed with the following primary antibodies overnight at $4^{\circ} \mathrm{C}$ in $2 \%(\mathrm{w} / \mathrm{v})$ BSA/TBS-T: human monoclonal antibody 6C5 [total tau (amino acids 125 to 131); 1:2000; Biogen] and human monoclonal antibody 40E8 (pS202-pT205 tau; 1:2000; Biogen). After washing three times in TBS-T, blots were incubated with horseradish peroxidase-conjugated goat antihuman (627120; Invitrogen, Carlsbad, CA) secondary antibody (1:4000 dilution in 2\% BSA/TBS-T) for 1 hour at room temperature. Immunoreactive proteins were developed using an enhanced chemiluminescence kit (Western Lightning; PerkinElmer, Waltham, MA) and captured on Hyperfilm ECL (GE Healthcare). Scanned images were analyzed using ImageJ version 1.48v (NIH, Bethesda, MD; http://imagej.nih. gov/ij) for densitometry quantification. 


\section{Tau ELISA}

The concentrations of human tau in the samples (brain extracts and SEC-separated fractions) were determined by Tau (total) Human ELISA kit (KHB0041; Life Technologies) and Tau (pS396) Human ELISA kit (KHB7031; Life Technologies), according to the manufacturer's instructions.

\section{AT8 ELISA}

AT8 (pS202/pT205-tau) ELISA assay was performed as described previously, ${ }^{12,25}$ with some modifications. Briefly, a 96-well plate (442404; Nunc MaxiSorp) was precoated with $2 \mu \mathrm{g} / \mathrm{mL}$ AT8 antibody (MN1020; $50 \mu \mathrm{L} /$ well; Thermo Scientific) in PBS overnight at $4^{\circ} \mathrm{C}$. The plate was then washed with PBS-T five times and blocked with 5\% BSA/ PBS for 1 hour at $37^{\circ} \mathrm{C}$. After washing with PBS-T three times, standards and samples were loaded onto the plate $\left(50 \mu \mathrm{L} /\right.$ well) and incubated overnight at $4^{\circ} \mathrm{C}$. Next, it was washed with PBS-T five times. Then, biotinylated-HT7 (MN1000B; 1:200 dilution in 1\% BSA/PBS; $50 \mu \mathrm{L} / \mathrm{well}$; Thermo Scientific) was added and incubated at room temperature for 1.5 hours, followed by five TBS-T washes. Streptavidin-poly-HRP40 (65R-S113; 1:4000 dilution in 1\% BSA/PBS; $50 \mu \mathrm{L} /$ well; Fitzgerald) was added to the plate and incubated at room temperature for 1 hour. After washing with PBS-T five times, the plate was developed by incubating with 1-step Ultra TMB-ELISA Substrate solution (34028; Thermo Scientific) for 20 minutes. The reaction was stopped by adding $2 \mathrm{~N} \mathrm{H}_{2} \mathrm{SO}_{4}$ and the plate was read at 450 $\mathrm{nm}$. For standard curves, an rTg4510 mouse brain homogenate (11-month-old, PBS-10,000 $\times g$ extract) at $100 \mu \mathrm{g} / \mathrm{mL}$ total protein was serially diluted with $1 \%$ BSA/PBS. The quantity of AT8-reactive (pS202/pT205) tau was determined using the standard curve and plotted as a relative amount of $\mathrm{rTg} 4510$ brain homogenates.

\section{Tau-Uptake Blocking Experiment}

6C5 tau antibody or control $\operatorname{IgG}$ (diluted with culture medium to $50 \mu \mathrm{g} / \mathrm{mL}$ ) was mixed with $\mathrm{rTg} 4510$ brain extract $(25 \mu \mathrm{g} / \mathrm{mL}$ total protein) at a 1:1 ratio (final, $25 \mu \mathrm{g} /$ $\mathrm{mL}$ antibody and $12.5 \mu \mathrm{g} / \mathrm{mL}$ total protein) and incubated for 30 minutes at room temperature. Then, $40 \mu \mathrm{L} /$ well $(0.5 \mu \mathrm{g}$ total protein/well) of the incubated sample was added directly to the primary neurons transduced with lentivirus. Quantification of neuronal tau uptake was performed 2 days after the treatment.

To evaluate whether tau antibody can retard the progression of tau uptake, rTg4510 brain extract (PBS$10,000 \times g$ ) was diluted with culture medium to make a final concentration of $16.7 \mu \mathrm{g} / \mathrm{mL}$ total protein and added to primary neurons on DIV $6(30 \mu \mathrm{L} /$ well, $0.5 \mu \mathrm{g}$ total protein/well). The following day (day 1), $30 \mu \mathrm{L} /$ well of $6 \mathrm{C} 5$ or $50 \mu \mathrm{g} / \mathrm{mL}$ control $\mathrm{IgG}$ was added to each well (final, $25 \mu \mathrm{g} / \mathrm{mL}$ antibody). Quantification of neuronal tau uptake was performed as above a day after the antibody treatment (day 2).

\section{In Vitro Tau Propagation Assay}

An interneuronal tau propagation assay was performed in a three-chamber microfluidic device, as described previously, ${ }^{4}$ with the following modifications (Supplemental Figure S1): Mouse primary neurons were loaded into the first and second chamber at an approximate density of $0.9 \times 10^{5}$ cells (in $10-\mu \mathrm{L}$ culture medium) and $0.24 \times 10^{5}$ cells (in $3-\mu \mathrm{L}$ culture medium), respectively. Neurons in the second chamber were transduced with lentivirus encoding tau RD P301L-CFP and tau RD P301L-YFP by adding $10 \mu \mathrm{L}$ of lentiviral vectors $(300 \mathrm{ng} / \mathrm{mL})$ to the second chamber on DIV2. Diffusion of lentiviral vectors from the second to the first chamber was prevented by a hydrostatic pressure barrier generated by adding $20 \mu \mathrm{L}$ of normal culture media to the first chamber. No CFP- or YFP-positive neurons were found in the first chamber on DIV 7, confirming that a hydrostatic pressure barrier was successful. The $\mathrm{rTg} 4510$ brain extract $(50 \mu \mathrm{g} / \mathrm{mL}$ total protein) was added to the first chamber ( $10 \mu \mathrm{L}$ in total) on DIV 7 . On the same day, $100 \mu \mathrm{g} / \mathrm{mL} 6 \mathrm{C} 5$ or control $\mathrm{IgG}$ antibody was added to the second chamber (30 $\mu \mathrm{L}$ in total) (DIV 7). The

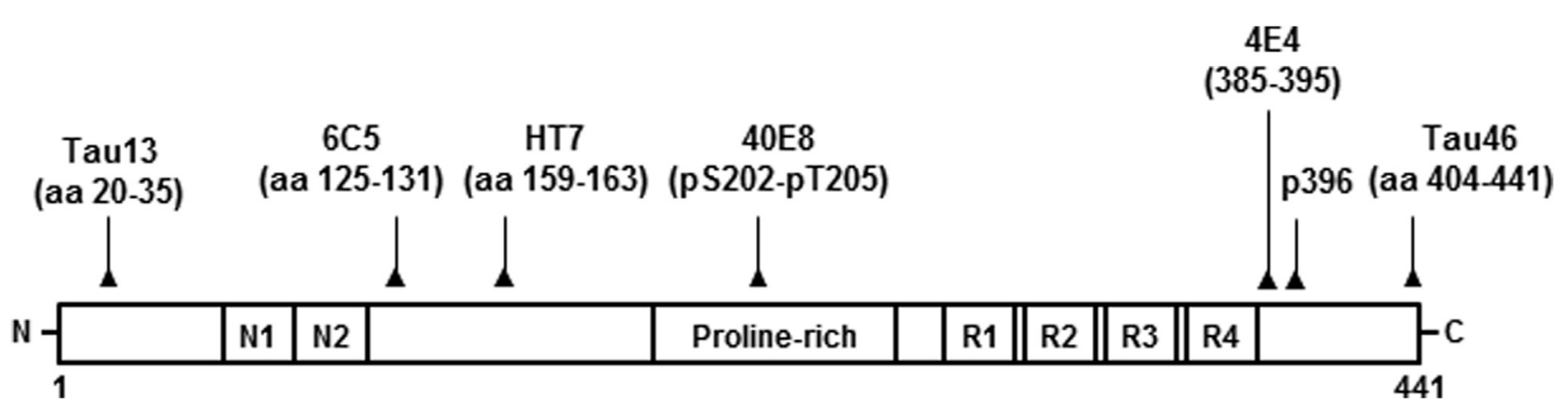

Figure 1 Tau antibodies used in the study. A schematic representation of the 441 amino acid (aa) isoforms of the human tau protein and the defined epitopes recognized by each tau antibody. Tau13, 6C5, HT7, 4E4, and Tau46 are phosphorylation-independent antibodies. 40 E8 and p396 are phosphorylation-dependent antibodies. C, carboxy-terminal; N, amino-terminal; N1-2, two inserts of 29 amino acids at the N-terminal part (exon 2 and 3); R1-4, microtubule-binding repeats. 
A

Total tau ELISA

(immunodepletion)

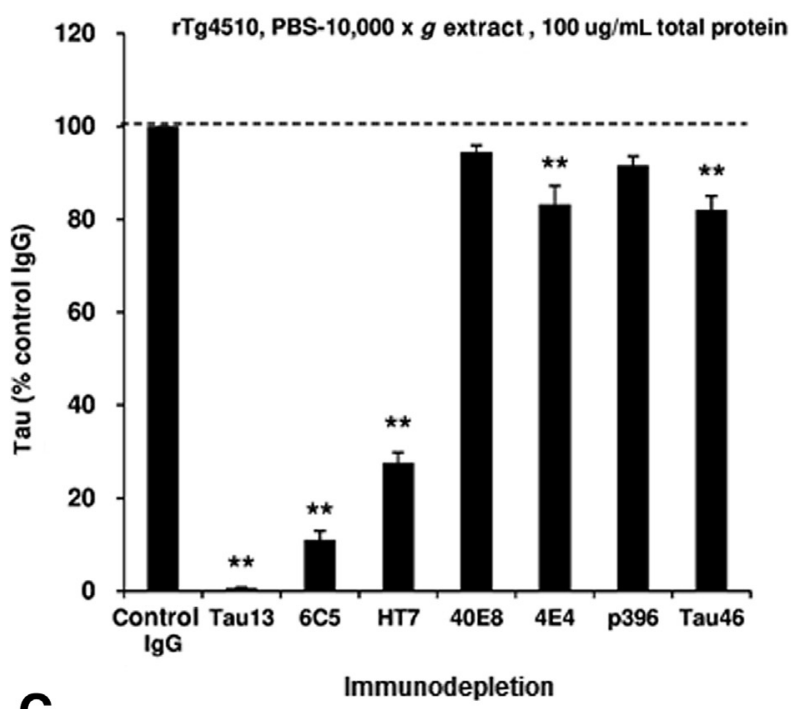

B

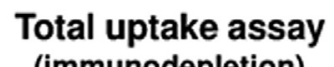

(immunodepletion)

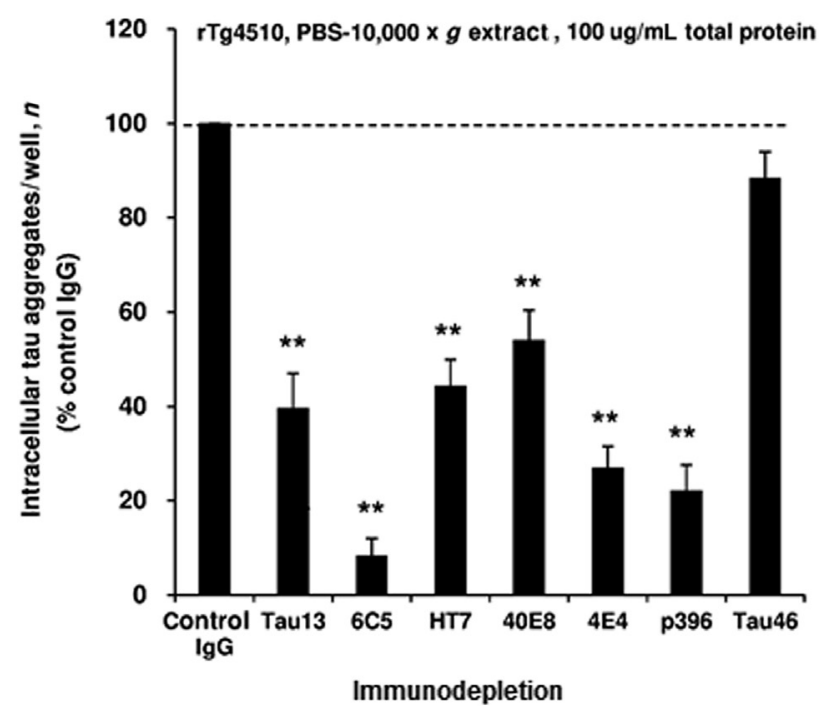

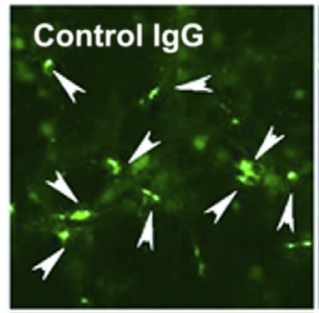
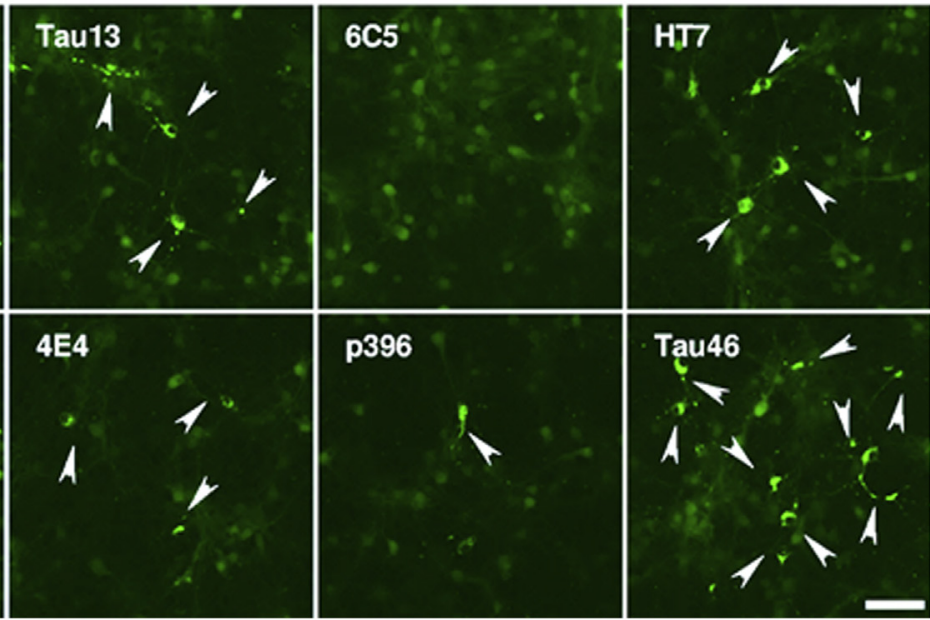

Figure 2 Tau uptake assay in primary neurons using tau-immunodepleted rTg4510 brain extract. A: rTg4510 brain extracts (11 to 13 months old; PBS-10,000 $\times g$ ) were immunodepleted using tau antibodies and total tau levels were measured by enzyme-linked immunosorbent assay (ELISA). 0ne-way analysis of variance with post hoc Dunnett multiple comparisons test was used. B and C: Tau uptake assay in primary neurons. Mouse primary neurons were transduced with lentivirus encoding tau repeat domain (RD) P301L-CFP and tau RD P301L-YFP on DIV 1 and treated with tau-immunodepleted rTg4510 brain extracts on DIV 6. The number of intracellular tau aggregates was quantified 2 days after treatment. One-way analysis of variance with post hoc Dunnett multiple comparisons test was used. Dashed lines represent control reference value (100\%). C: Representative confocal images (FRET channel) of tau uptake in primary neurons. Arrowheads indicate intracellular tau aggregates. $n=5$ per group $(\mathbf{A}-\mathbf{C}) .{ }^{*} P<0.01$ versus control IgG. Scale bar $=20 \mu \mathrm{m}(\mathbf{C})$.

number of tau-aggregate positive neurons in the second chamber was quantified 6 days after the treatment (DIV 13).

\section{Statistical Analysis}

All data were expressed as means \pm SEM. Two-group comparisons were performed by two-tailed $t$-tests. Comparison among three or more groups was performed by oneway analysis of variance and post hoc Dunnett multiple comparison test. For comparing two different treatments over time, a two-way repeated measures analysis of variance with post hoc Sidak multiple corrections test was used. The statistical test used is described in each figure legend. $P<0.05$ was considered significant.

\section{Results}

Production and Characterization of Novel Antibodies against Human Tau

Figure 1 and Table 2 summarize the tau antibodies used in this study, their target epitopes, and their affinities 
A

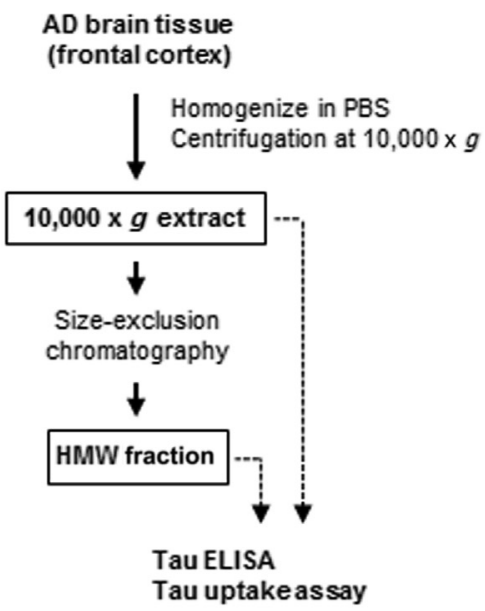

B

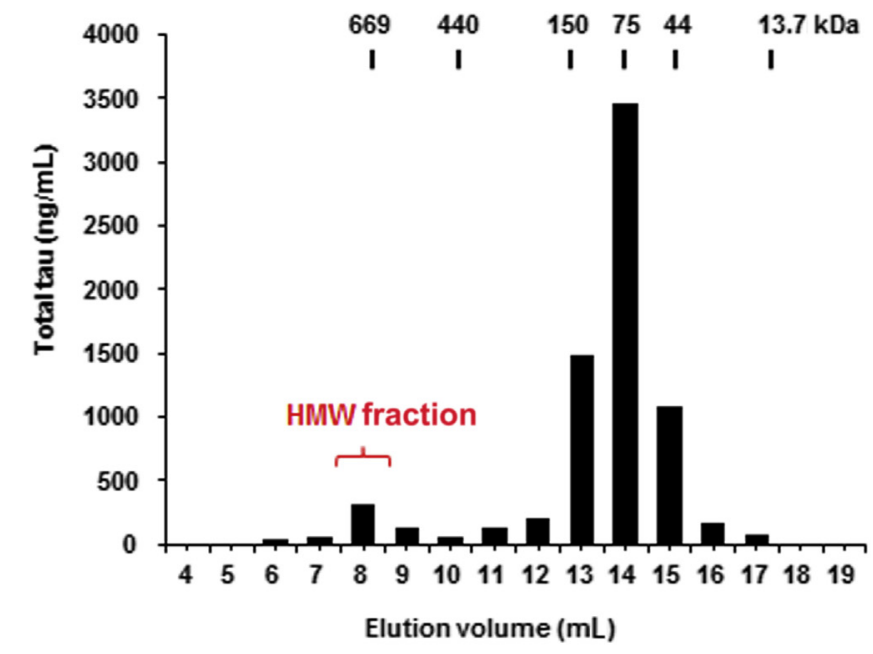

C

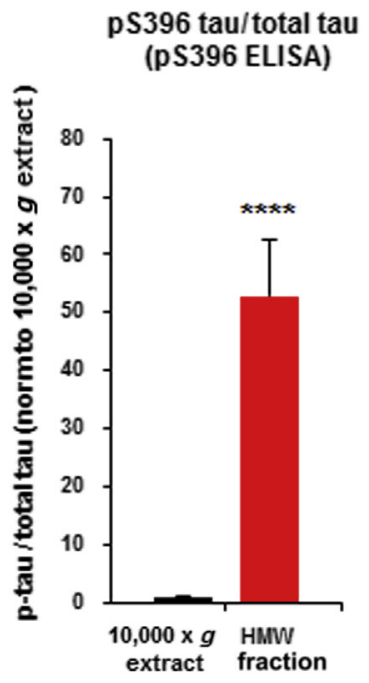

pS202/pT205 tau/total tau (AT8 ELISA)

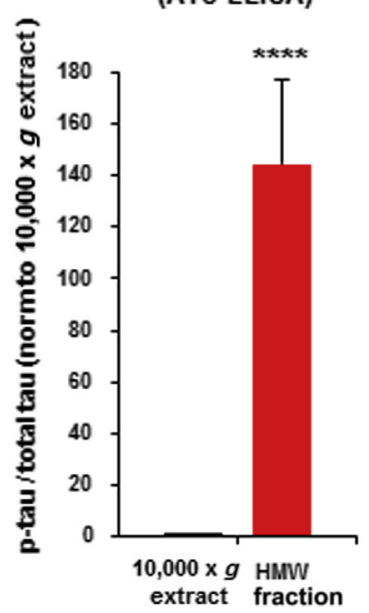

D
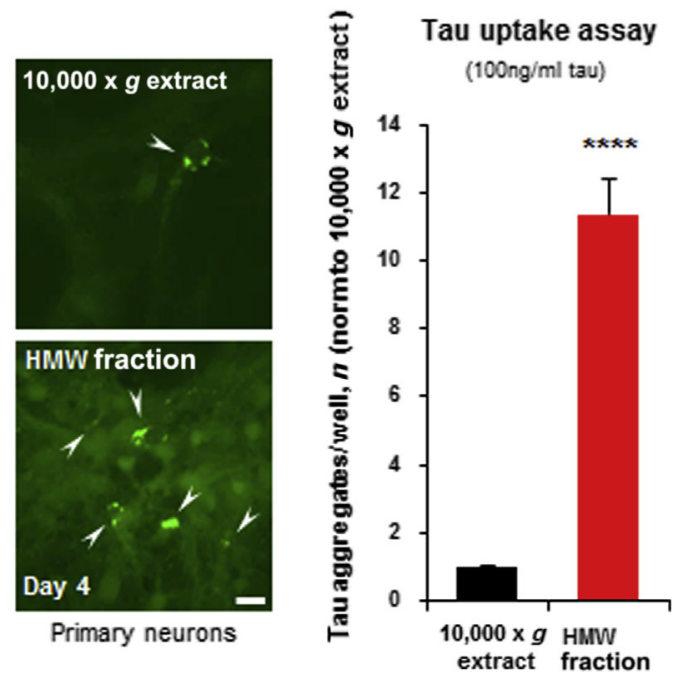

Figure 3 High-molecular-weight (HMW) tau species isolated from human AD brain extract is highly phosphorylated and readily taken up by neurons. A: Schematic of the protocol for the preparation of PBS-soluble 10,000 $\times g$ extract from AD postmortem brain tissue and the isolation of HMW tau species by size-exclusion chromatography (SEC). B: Representative graph of tau levels in SEC-separated AD brain extract (PBS-10 kg). Total tau levels in each SEC fraction were measured using total tau enzyme-linked immunosorbent assay (ELISA). C: Quantification of phospho-tau levels in $10,000 \times g$ extract and HMW fraction (pS396 and AT8 ELISAs). Two-tailed $t$-test was used. D: Tau uptake assay in primary neurons. Mouse primary neurons were transduced with lentivirus encoding tau RD P301L-CFP and tau RD P301L-YFP on DIV 1 and treated with AD brain $10 \mathrm{~kg}$ extract or HMW fraction on DIV 6. The number of intracellular tau aggregates was quantified 4 days after treatment. D: Representative confocal images (FRET channel). Arrowheads indicate intracellular tau aggregates. Quantification of tau uptake on day $4 . n=4$ per group (C and $\mathbf{D}$ ). ${ }^{* * * *} P<0.0001$ versus $10,000 \times g$ (two-tailed t-test). Scale bar $=20 \mu \mathrm{m}$.

for recombinant nonphosphorylated tau or for $\mathrm{AD}$ brain-derived paired helical filament tau. Along with several widely available antibodies targeting N-terminal (Tau13), middomain (HT7), C-terminal end (Tau46), and phosphorylated (anti-pS396) epitopes of tau, we evaluated three novel human antibodies derived by recombinant cloning from human B cells, targeting residues 125 to 131 (clone 6C5), 385 to 395 (clone 4E4), and pS202/pT205 (clone 40E8).
Tau Antibodies Have Distinct Effects on Neuronal Tau Uptake from rTg4510 Brain Extract

To study the effects of tau antibodies on neuronal tau uptake, we used a sensitive FRET-based assay in mouse primary neurons. Neurons were transduced with lentivirus encoding tau RD with P301L mutation tagged with CFP or YFP, which produce a strong FRET signal on tau uptake and subsequent intracellular aggregation. We used 

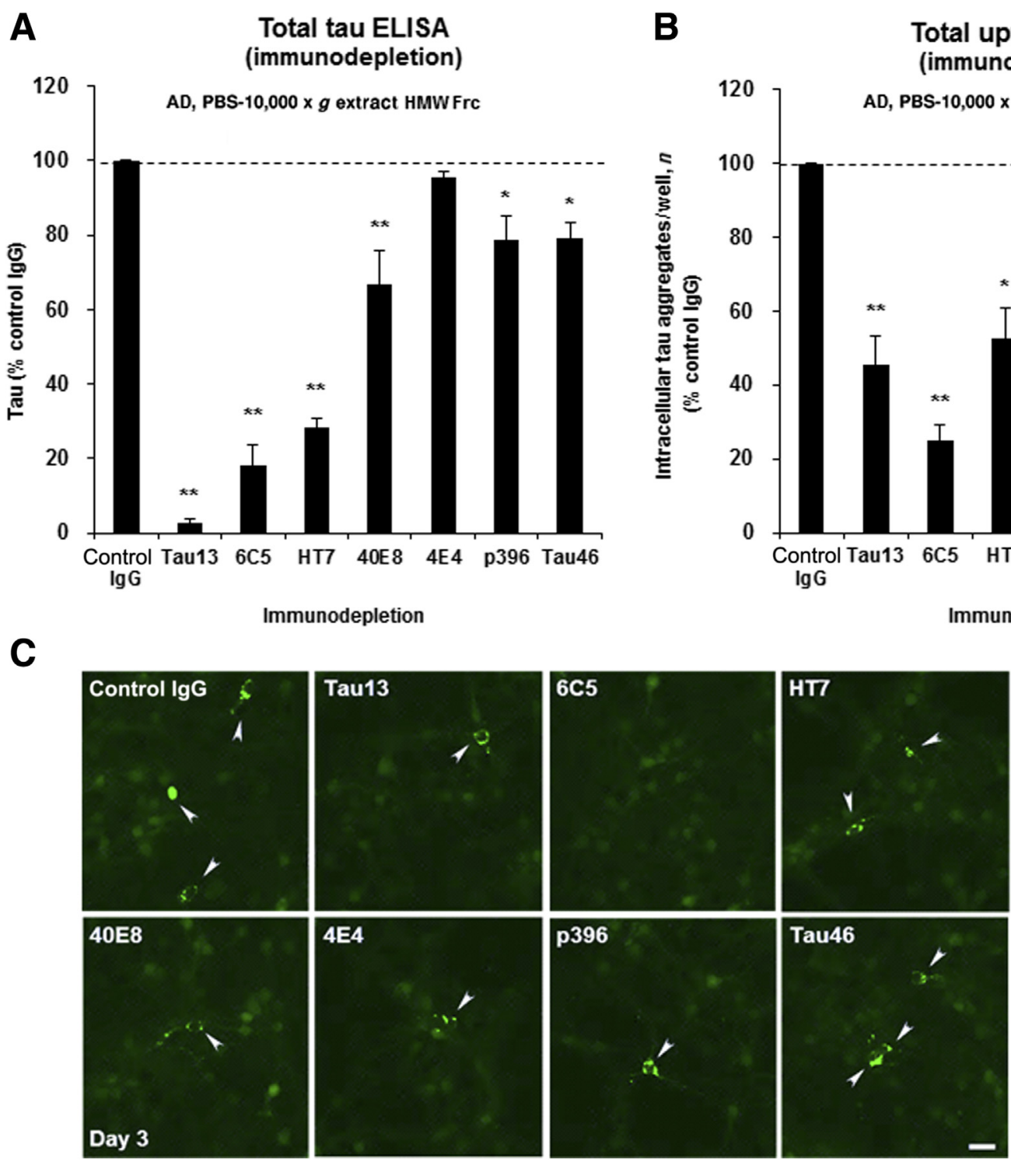

Figure 4 Tau uptake assay in primary neurons using tau-immunodepleted human AD brain extract. A: High-molecular-weight (HMW) fraction isolated from human AD brain extract (PBS-10,000 $\times g$ ) was immunodepleted using tau antibodies, and total tau levels were measured by enzyme-linked immunosorbent assay (ELISA). One-way analysis of variance with post hoc Dunnett multiple comparisons test was used. B and C: Tau uptake assay in primary neurons. Mouse primary neurons were transduced with lentivirus encoding tau repeat domain (RD) P301L-CFP and tau RD P301L-YFP on DIV 1 and treated with tau-immunodepleted HMW fraction of AD brain extracts on DIV 6. The number of intracellular tau aggregates was quantified 3 days after treatment. One-way analysis of variance with post hoc Dunnett multiple comparisons test was used. Dashed lines represent control reference value (100\%). C: Representative confocal images (FRET channel) of tau uptake in primary neurons. Arrowheads indicate intracellular tau aggregates. $n=4$ per group (A and B). ${ }^{*} P<0.05$, ${ }^{* *} P<0.01$ versus control IgG. Scale bar $=20 \mu \mathrm{m}$ (C).

PBS-soluble low-spin $(10,000 \times g)$ extracts of $\mathrm{rTg} 4510$ brain (11 to 13 months old) containing bioactive tau species involved in neuronal uptake ${ }^{4}$ to treat the neurons after immunodepletion (Figure 2).

Among the seven antibodies, Tau13 and 6C5 most efficiently removed tau ( $>85 \%$ reduction) from $\mathrm{rTg} 4510$ brain extracts on immunodepletion (Figure 2A). HT7 showed an intermediate effect (72\% reduction), whereas the other four antibodies (40E8, 4E4, p396, and Tau46) removed only a small fraction of tau $(5.6 \%, 16.6 \%, 8.4 \%$, and $18 \%$ reductions, respectively) (Figure $2 \mathrm{~A}$ ). We then assessed tau uptake from the tau-immunodepleted brain extract in primary neurons (Figure 2, B and C). Confocal FRET image analysis showed robust tau aggregation in primary neurons treated with control IgG-immunodepleted $\mathrm{rTg} 4510$ brain extracts (Figure 2, B and C). The 6C5 antibody most successfully reduced tau uptake by immunodepletion $(>90 \%$ reduction), and Tau13 and HT7 showed intermediate effects 
Uptake levels: \% inhibition (antibodies are listed from best to worst)

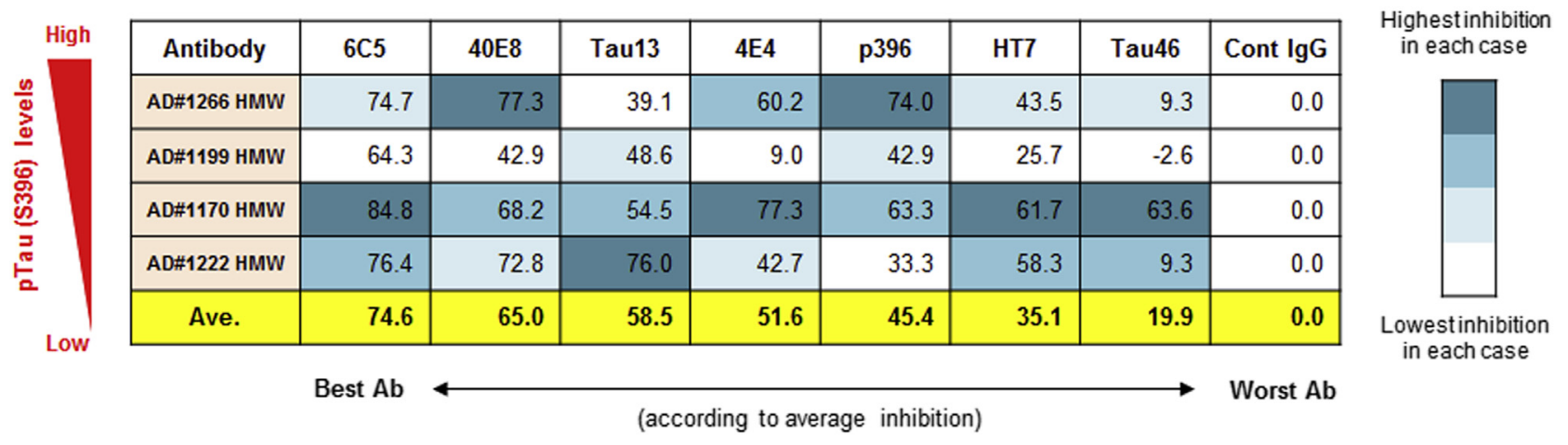
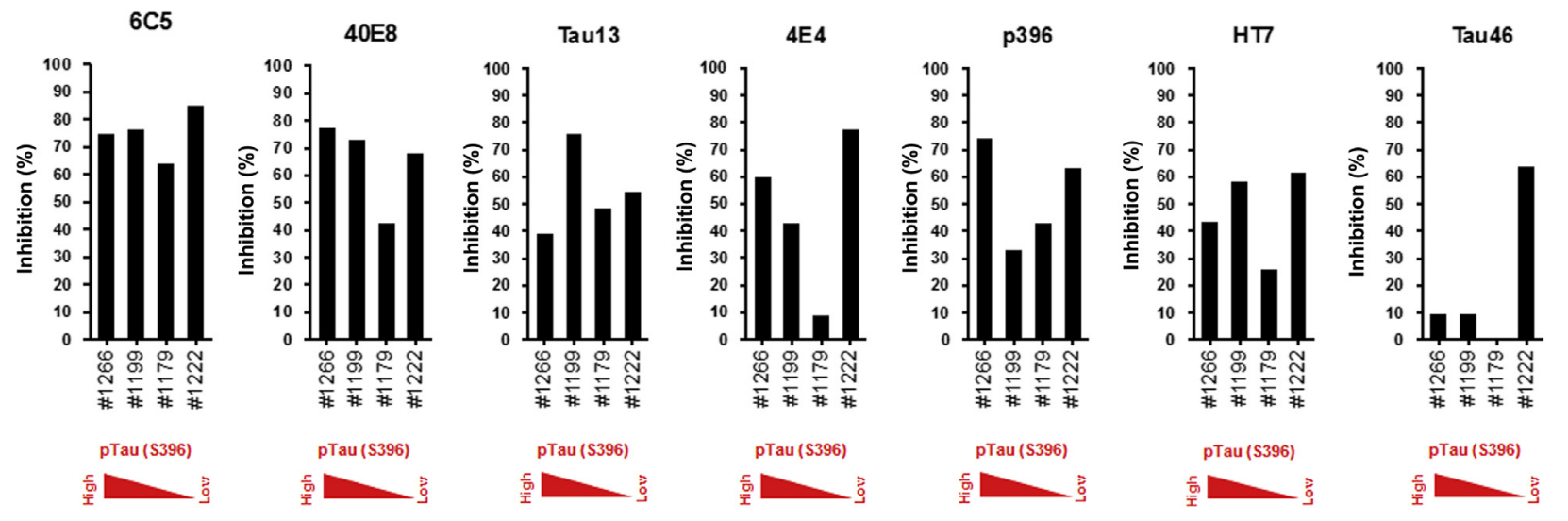

Figure 5 Antibody efficacy based on percentage inhibition of tau uptake varies by case. Phospho-tau (pS396) levels in size-exclusion chromatography (SEC)-separated high-molecular-weight (HMW) fraction of human AD brain were measured using enzyme-linked immunosorbent assay (ELISA). Cases were ordered by decreasing level of phospho-tau $(1266,1199,1170,1222)$ and plotted against each antibody. Percentage inhibition was based on the reduction in the number of intracellular tau aggregates for antibody-depleted versus control IgG. Ab, antibody.

(approximately 60\% reductions) (Figure 2, B and C). The 40E8, 4E4, and p396 antibodies also reduced neuronal tau uptake by $40 \%$ to $80 \%$, despite their low-immunodepletion efficiency (Figure 2). This finding suggests that they interacted with tau species that are prone to cellular uptake, which account for only a small fraction of all soluble tau species in the brain extract. Notably, Tau46 had little effect on tau uptake (Figure 2, B and C).
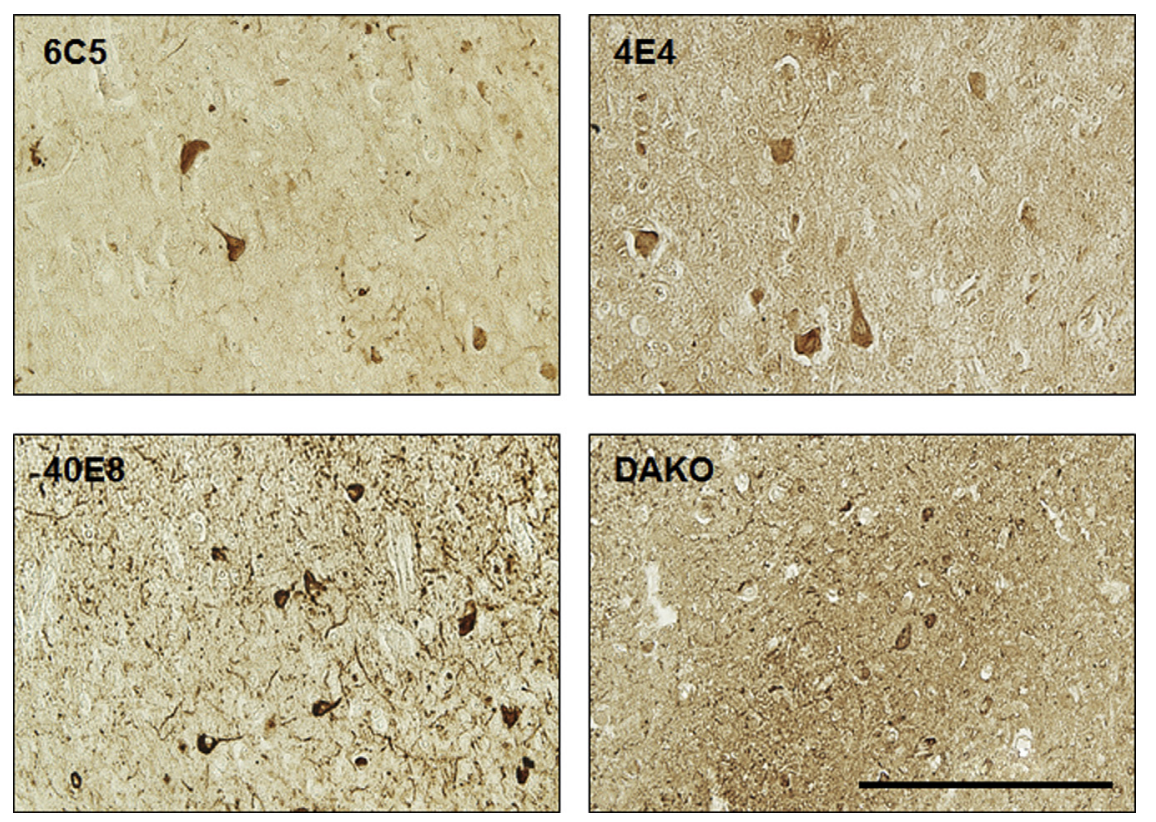

Figure 6 Tau antibodies immunostain NFTs in postmortem human $A D$ brain. Frontal cortex sections from human $A D$ brain (1266) were immunostained with primary human monoclonal antibodies: phosphorylation-independent tau antibodies $6 \mathrm{C} 5$ and 4E4, and phosphorylationdependent tau antibody 40E8. Rabbit polyclonal anti-tau antibody (Dako) served as a positive control. All tau antibodies showed positive NFT staining. Scale bar $=200 \mu \mathrm{m}$. 


\section{A Tau uptake assay (rTg4510, PBS-10,000 x $g$ extract + antibody)}

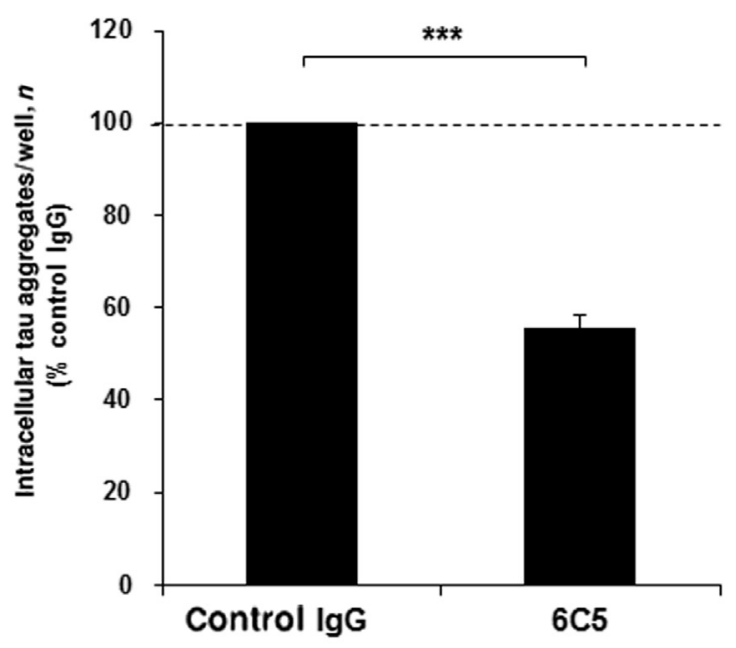

B
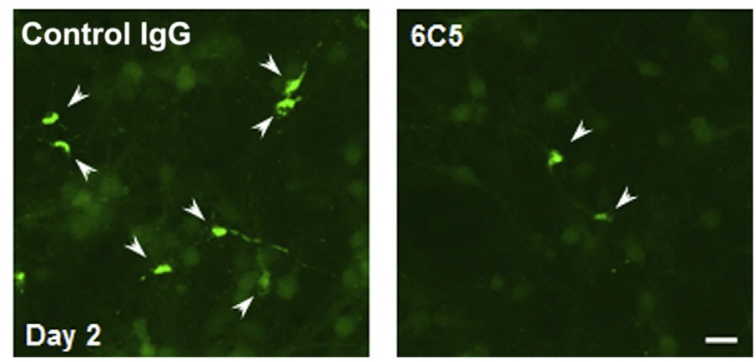

Figure 7 Tau antibody $6 \mathrm{C} 5$ blocks neuronal tau uptake without immunodepletion. A: $6 \mathrm{C} 5$ or control IgG (final, $25 \mu \mathrm{g} / \mathrm{mL}$ ) antibody was added to rTg4510 brain extracts (11 to 13 months old; PBS-10,000 $\times$ g; final, $12.5 \mu \mathrm{g} / \mathrm{mL}$ total protein) and incubated for 30 minutes at room temperature. Subsequently, the incubated samples were added to mouse primary neurons that were transduced with lentivirus encoding tau repeat domain (RD) P301L-CFP and tau RD P301L-YFP. The number of intracellular tau aggregates was quantified 2 days after treatment. Two-tailed $t$-test was used. Dashed line indicates control reference value (100\%). B: Representative confocal images (FRET channel) of tau uptake in primary neurons. Arrowheads indicate intracellular tau aggregates. $n=7$ per group (A). $* * * P<0.001$. Scale bar $=20 \mu \mathrm{m}$.

Hyperphosphorylated HMW Tau from Human Alzheimer Brain Is Readily Taken Up by Neurons

We next assessed neuronal tau uptake using pathologically relevant HMW tau species extracted from human $\mathrm{AD}$ brains, species that are readily taken up by neurons ${ }^{4}$ (Figure 3). Postmortem brain tissues (frontal cortex) were obtained from four cases of neuropathologically confirmed AD patients (all Braak stage VI) (Table 1). We fractionated PBS-soluble brain extracts $(10,000 \times g$ spin $)$ by SEC (Figure 3A), and collected a small fraction comprising HMW tau (Figure 3B). The isolated HMW tau was highly phosphorylated (Figure 3C) and more bioactive in terms of tau uptake (by 11 times) (Figure 3D) compared to the original $10,000 \times g$ spin extract.
Tau Antibodies Have Distinct Effects on Neuronal Tau Uptake of HMW Tau Derived from Human AD Brain

Similar to the results of experiments using $\mathrm{rTg} 4510$ brain (Figure 2A), Tau13, 6C5, and HT7 efficiently depleted tau from the $\mathrm{AD} \mathrm{HMW}$ fraction $(97 \%, 82 \%$, and $72 \%$, respectively), whereas the other four antibodies $(40 \mathrm{E} 8,4 \mathrm{E} 4$, p396, and Tau46) removed only a small fraction of tau (33\%, 4.7\%, 22\%, and $21 \%$ reductions, respectively) (Figure 4A). In the tau uptake assay, 6C5 most effectively reduced tau uptake by immunodepletion (75\% reduction) (Figure 4, B and C). Tau13 and HT7 showed intermediate effects (55\% and 47\% reductions, respectively) (Figure 4, B and C). The 40E8, p396, and 4E4 antibodies also reduced neuronal tau uptake $(65 \%, 53 \%$, and $47 \%$ reductions, respectively), despite their low immunodepletion efficiency (Figure 4). This finding suggests that 40E8, p396, and 4E4 specifically target lower abundance tau species important for uptake in the HMW fraction. SDS-PAGE and Western blot analysis of 40E8- and 6C5-immunodepleted supernatants showed that although the phosphorylation-dependent antibody 40E8 removed a small fraction of total tau, 40E8 depleted a significant fraction (67\%) of phosphorylated tau (Supplemental Figure S2). Notably, and similar to the $\mathrm{Tg} 4510$ uptake results (Figure 2B), Tau46 was the only antibody that did not show a statistically significant reduction in neuronal tau uptake (Figure 4, B and C). Tau46 bound with high apparent affinity to both recombinant and paired helical filament tau by ELISA (Table 2), demonstrating the binding of the antibody to full-length tau.

Interestingly, we observed a variation in the blocking efficiency (percentage inhibition compared to control IgG) for each tau antibody among the various $\mathrm{AD}$ cases tested (Figure 5), implying that the species composition of tau might differ among patients; for example, the two phosphorylationdependent tau antibodies (40E8 and p396) were the most efficient in the human $\mathrm{AD}$ case with the highest level of phosphorylated tau (1266). Both 6C5 and 40E8, shown to be most effective at reducing uptake from HMW human $\mathrm{AD}$ brain-derived tau species (Figure 3, B and C), immunostained NFTs and neuritic plaques in postmortem human $\mathrm{AD}$ frontal cortex sections (Figure 6); 40E8 was somewhat more reactive to neuropil threads under the conditions used.

\section{C5 Tau Antibody Directly Blocks Neuronal Tau Uptake and Interneuron Propagation}

We next examined if a tau antibody can directly block tau uptake into neurons. In the following experiments, we used the $6 \mathrm{C} 5$ antibody because it most efficiently depleted the tau species involved in neuronal uptake (Figures 2 and 4) and we chose to use rTg4510 brain extract because of an overall higher propensity to seed as compared to human sporadic $\mathrm{AD}$ brain extract. This allowed us to test the efficiency of the 6C5 antibody to block tau uptake and propagation without immunodepletion (Figures 7, 8, and 9). The 6C5 or 
A

3-chamber microfluidic device

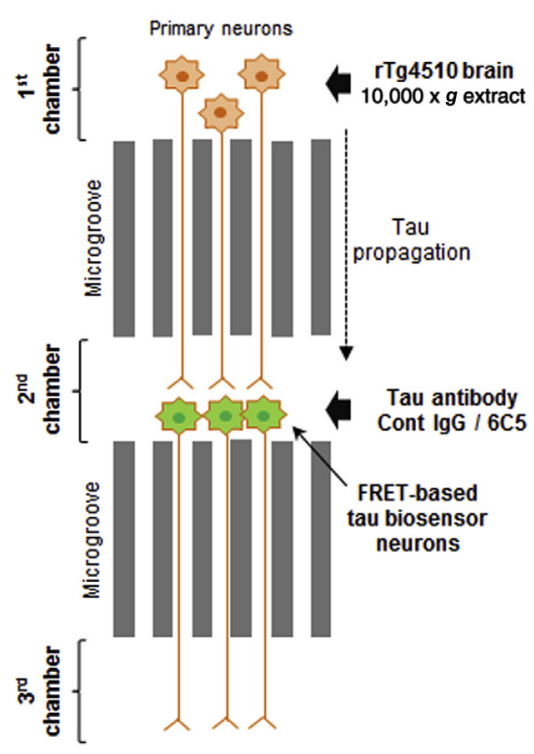

B
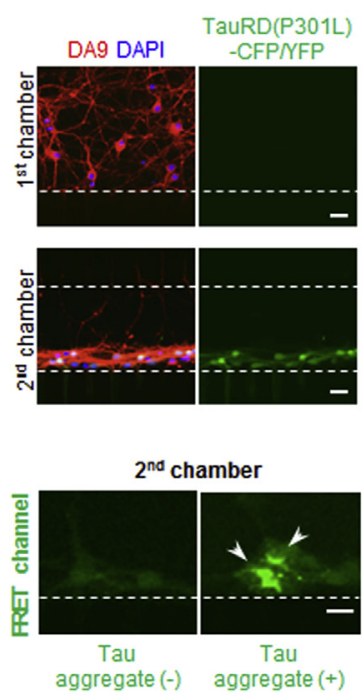

C

Tau propagation in the 2nd chamber
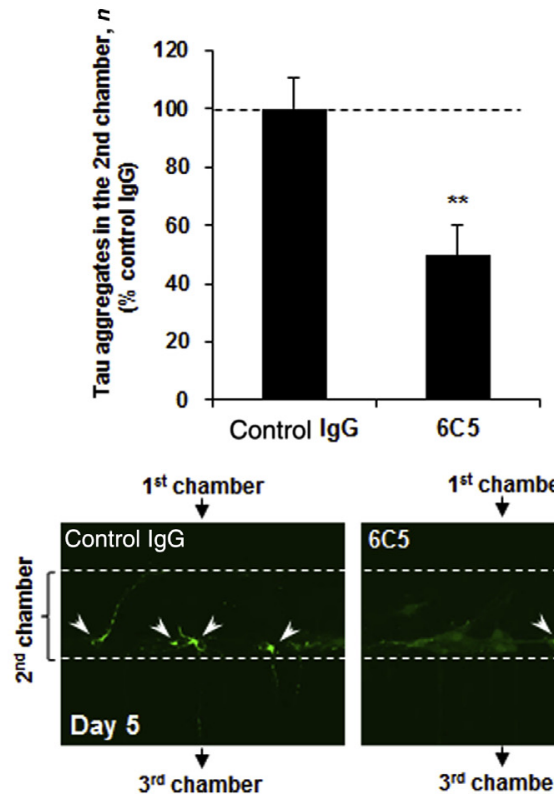

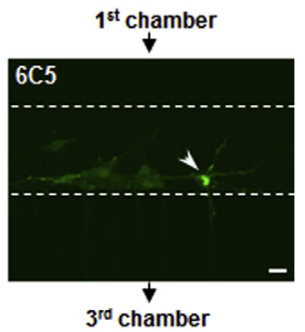

Figure 8 Tau antibody 6 C5 blocks neuron-to-neuron tau spreading in a three-chambered microfluidic device. A: Schematic of a three-chambered microfluidic device for culturing primary neurons. Neurons in the second chamber were transduced with lentivirus encoding tau repeat domain (RD) P301L-CFP and tau RD P301L-YFP to detect propagated tau from the first chamber neurons. rTg4510 brain extracts (11 to 13 months old) were added to the first chamber. The number of intracellular tau aggregates in the second chamber neurons was quantified 5 days after treatment. B: Representative images of neurons in the first and second chambers stained with total-tau antibody DA9 (red, as the neuronal marker) and DAPI (blue). Expression of lentiviral-induced proteins (P301L-CFP and P301L-YFP) in the second chamber neurons is shown in green. Dashed lines represent distinct chambers. Representative confocal images (FRET channel) of the second chamber neurons at day 5 . The second chamber neurons that received pathological tau from the first chamber neurons develop FRET-positive intracellular tau aggregates (arrowheads). C: Quantification of the number of tau aggregates in the second chamber neurons (tau propagation). Two-tailed $t$-test was used. Black dashed line indicates control reference value $(100 \%)$. White dashed lines represent distinct chambers. Representative confocal images (FRET channel) of tau uptake in the second chamber neurons are also shown (bottom row). Arrowheads indicate intracellular tau aggregates. $n=10$ to 11 devices per group (C). ${ }^{* *} P<0.01$ versus control IgG (two-tailed t-test). Scale bars $=20 \mu \mathrm{m}$.

control IgG antibody was incubated with rTg4510 brain extracts and then directly added to primary neurons. At day 2, 6C5 significantly reduced neuronal tau uptake by $44 \%$ compared to control IgG (Figure 7).

Next, we tested if the antibody can block neuron-to-neuron propagation by using a unique microfluidic neuron device (Figure 8 and Supplemental Figure S1). The device is composed of three distinct chambers connected through microgrooves. Two sets of primary neurons in the first and second chambers have in-line synaptic connections at the second chamber. ${ }^{4}$ We transduced the second chamber neurons with lentivirus encoding tau RD P301L-CFP and tau RD P301L-YFP, so they could sensitively detect tau transmitted from the first chamber neurons (Figure 8). We treated the first chamber neurons with rTg4510 brain extracts (PBS$10,000 \times g)$ and quantified the number of tau aggregate-positive neurons in the second chamber 6 days after the treatment. $6 \mathrm{C} 5$ or control $\mathrm{IgG}$ antibody was added to the second chamber to test the hypothesis that antibodies could block tau propagation from the first to second chamber neurons, presumably reflecting release of tau seeds from the axons of neurons in the first chamber and uptake/aggregation into the biosensor neurons in the second chamber. The microfluidic design precluded simple diffusion of tau seeds from the first chamber to the second chamber. The number of tau-aggregate positive neurons in the second chamber was significantly lower in the 6C5-treated neurons than in the control IgG (Figure 8), suggesting that $6 \mathrm{C} 5$ can block the interneuron propagation of tau.

Furthermore, we examined whether the $6 \mathrm{C} 5$ antibody can slow down the progression of tau pathology: 6C5 or control IgG antibody was added to the neuron culture a day after the initiation of the tau-seed treatment (rTg4510 brain extract). The number of tau aggregate-positive neurons increases over time; however, treatment with $6 \mathrm{C} 5$ antibody had significantly less evidence of tau uptake by day 2 (a day after initiation of the antibody treatment) as compared to the control IgG-treated neurons (Figure 9). This result is consistent with the idea that the 6C5 antibody can slow tau uptake even after neurons have been exposed to the pathological tau and the uptake process initiated.

\section{Discussion}

The progression of tau pathology in a hierarchical pattern is tightly linked with the clinical progression of patients with $\mathrm{AD}^{2,3}$ It is therefore possible that blocking tau species 


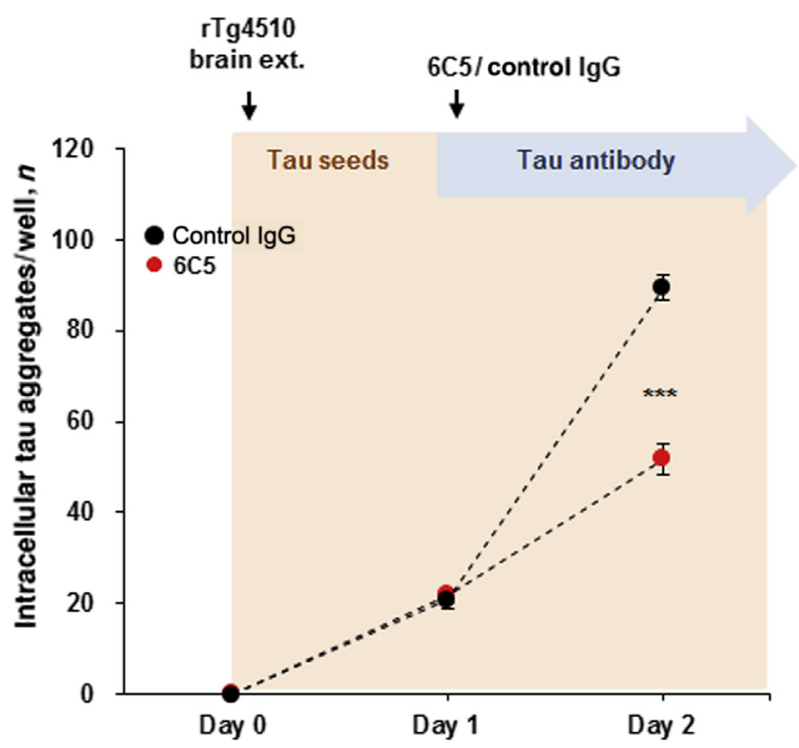

Figure 9 Tau antibody $6 \mathrm{C} 5$ slows down the progression of neuronal tau uptake. rTg4510 brain extract (11 to 13 months old; PBS-10,000 $\times g$ ) were added to mouse primary neurons that were transduced with lentivirus encoding tau RD P301L-CFP and tau RD P301L-YFP on day 0. Addition of tau seeds to culture is represented by orange shading. The following day, 6C5 or a control IgG antibody was added to the culture dish, represented by a blue arrow. The number of intracellular tau aggregates was quantified i) before adding $\mathrm{rTg} 4510$ brain extracts (day 0), ii) before adding antibodies (day 1), and iii) a day after the antibody treatment (day 2). Two-way repeated measures analysis of variance with post hoc Sidak multiple comparisons correction was used. $n=6$ per group. ${ }^{* *} P<0.001$ versus control IgG (two-way repeated measures analysis of variance with post hoc Sidak multiple comparisons correction).

involved in interneuron propagation may affect disease progression. This study aimed to test the effect of a panel of tau antibodies on neuronal tau uptake in vitro and to identify suitable target epitopes and antibodies to efficiently block tau uptake and propagation. We used a sensitive FRETbased tau uptake assay in primary neuron culture; meanwhile, a unique microfluidic device allowed us to test the effect of tau antibody treatment on neuron-to-neuron propagation. We used relevant tau species derived from the brain extracts of tau-transgenic mice and human $\mathrm{AD}$ patients, species that can be taken up by neurons even without the aid of protein transfection reagents. In human AD brain, HMW species specifically were separated by SEC from PBS$10,000 \times g$ extracts to concentrate and isolate species with a high propensity for neuronal uptake ${ }^{4}$ (Figure 3D), because human $\mathrm{AD}$ lysate does not seed as strongly as rTg4510 lysate. We find that tau antibodies against distinct epitopes affected neuronal tau uptake in different ways.

We tested four phosphorylation-independent antibodies in this study; each recognizes N-terminal (Tau13), middomain (6C5 and HT7), and C-terminal (Tau46) epitopes of tau. Tau13, 6C5, and HT7 successfully captured tau species involved in neuronal uptake, with $6 \mathrm{C} 5$ being the most efficient at reducing uptake; however, Tau46 had little effect on uptake using both $\mathrm{rTg} 4510$ and human $\mathrm{AD}$ brain extracts (Figures 2 and 4). This finding suggests that the tau species involved in neuronal uptake and present in the PBS-soluble brain extract lack access to the C-terminal end, because of folding, post-translational modifications, or cleavage. Previous reports also demonstrate that soluble extracellular tau in human $\mathrm{AD}^{26}$ and tau-transgenic mouse cerebrospinal fluid ${ }^{19}$ is comprised primarily of various truncated forms, with most species not containing an intact C-terminal region. Other studies have reported that physiologically $^{27}$ and pathophysiologically secreted ${ }^{28}$ tau can exist in a full-length form, although their significance to neuronal uptake remains unclear. Therapeutic antibodies for blocking propagation must target an intact and accessible epitope; therefore, a far C-terminal epitope may not be optimal.

Mechanisms by which the therapeutic tau antibody clears tau pathology in vitro and in vivo remain largely unknown. The tau antibody may capture and neutralize extracellular pathological tau, preventing it from being taken up by neurons. The tau-antibody complex may be cleared by microglial cells after $\mathrm{Fc}-\gamma$ receptor-mediated phagocytosis. ${ }^{29}$ Alternatively, the antibody may be taken up by neurons, promoting intracellular degradation of pathological tau via the lysosomal pathway. ${ }^{30,31}$ In the present study, the 6C5 antibody directly inhibited neuronal tau uptake simply by being added to the culture medium (Figure 7). This finding implies that the antibody blocked at least one of the following processes: i) the binding of the extracellular tau to the cell surface, ii) the internalization of tau attached on the cell membrane into the cytoplasm, or iii) the subsequent intracellular aggregation. Future studies should elucidate on the mechanisms in greater detail. Notably, the mechanism of tau uptake into neurons is incompletely understood; further insights would likely improve our understanding of how antibodies reduce intracellular tau aggregation. Previous research has reported that tau antibodies can promote tau uptake into microglia via $\mathrm{Fc}$ receptors ${ }^{29}$; however, this mechanism is unlikely to be the major pathway in our assay because there were few, if any, microglia in our primary neuron culture.

More important, the 6C5 antibody affected neuronal tau aggregations even after the uptake process had been initiated (Figure 9). This is indirect evidence, in accord with transgenic animal data, that tau antibody treatment can be effective even after tau deposition has started. ${ }^{9,16,32-34}$ This result is of particular importance considering the fact that tau pathology develops years before the onset of the dementia symptoms ${ }^{35}$ and a precise diagnosis of $\mathrm{AD}$ at an early stage is still challenging.

Phosphorylation-dependent tau antibodies 40E8 (requiring phosphorylation of serine 202 and threonine 205) and p396 (against phospho-serine 396) were less efficient at immunodepleting tau from the mixture of tau species present in the brain extracts; however, they efficiently reduced neuronal tau uptake (Figures 2 and 4). This finding suggests that most of the tau taken up by neurons is phosphorylated at least at these three sites. Targeting phospho-tau epitopes 
is likely to be a straightforward approach because tau is known to be hyperphosphorylated in the NFT, and indeed, some phospho-tau-specific tau antibodies are reported to reduce pathology and ameliorate cognitive impairment in animal models. ${ }^{10,12,14,15}$ We previously observed a relatively large variation in phosphorylation levels at each phospho-site in AD-derived tau. ${ }^{4}$ In one tau-transgenic mouse model, the phospho-tau antibodies specific to distinct phosphorylation sites affected tau pathology differently, with a certain phospho-tau antibody (PG5, pS409) even worsening the pathology. ${ }^{13}$ It will be important to screen multiple phosphorylation sites in the same in vitro and/or in vivo assay to identify the most efficient therapeutic antibody.

Our results imply that not all tau epitopes are equally robust in terms of blocking the neuronal uptake of tau derived from tau-transgenic mouse and the human $\mathrm{AD}$ brain. Notably, each AD case appears to have a somewhat distinct species composition of tau, which may affect the blocking efficiencies of certain tau antibodies (Figure 5). Identification and characterization of the biochemical signature of tau specifically involved in uptake and propagation will be necessary to develop an efficient therapeutic antibody targeting tau propagation.

\section{Acknowledgment}

We thank Dr. Feng Chen for her contributions toward the generation and characterization of the human-derived tau antibodies.

\section{Supplemental Data}

Supplemental material for this article can be found at http://dx.doi.org/10.1016/j.ajpath.2017.01.022.

\section{References}

1. Mandelkow EM, Biernat J, Drewes G, Gustke N, Trinczek B, Mandelkow E: Tau domains, phosphorylation, and interactions with microtubules. Neurobiol Aging 1995, 16:355-362; discussion 62-63

2. Braak H, Braak E: Neuropathological stageing of Alzheimer-related changes. Acta Neuropathol 1991, 82:239-259

3. Hyman BT, Van Hoesen GW, Damasio AR, Barnes CL: Alzheimer's disease: cell-specific pathology isolates the hippocampal formation. Science 1984, 225:1168-1170

4. Takeda S, Wegmann S, Cho H, DeVos SL, Commins C, Roe AD, Nicholls SB, Carlson GA, Pitstick R, Nobuhara CK, Costantino I, Frosch MP, Muller DJ, Irimia D, Hyman BT: Neuronal uptake and propagation of a rare phosphorylated high-molecular-weight tau derived from Alzheimer's disease brain. Nat Commun 2015, 6: 8490

5. de Calignon A, Polydoro M, Suarez-Calvet M, William C, Adamowicz DH, Kopeikina KJ, Pitstick R, Sahara N, Ashe KH, Carlson GA, Spires-Jones TL, Hyman BT: Propagation of tau pathology in a model of early Alzheimer's disease. Neuron 2012, 73 : 685-697
6. Liu L, Drouet V, Wu JW, Witter MP, Small SA, Clelland C, Duff K: Trans-synaptic spread of tau pathology in vivo. PLoS One 2012, 7:e31302

7. Harris JA, Koyama A, Maeda S, Ho K, Devidze N, Dubal DB, Yu GQ, Masliah E, Mucke L: Human P301L-mutant tau expression in mouse entorhinal-hippocampal network causes tau aggregation and presynaptic pathology but no cognitive deficits. PLoS One 2012, 7:e45881

8. Pedersen JT, Sigurdsson EM: Tau immunotherapy for Alzheimer's disease. Trends Mol Med 2015, 21:394-402

9. Yanamandra K, Kfoury N, Jiang H, Mahan TE, Ma S, Maloney SE, Wozniak DF, Diamond MI, Holtzman DM: Anti-tau antibodies that block tau aggregate seeding in vitro markedly decrease pathology and improve cognition in vivo. Neuron 2013, 80:402-414

10. Umeda T, Eguchi H, Kunori Y, Matsumoto Y, Taniguchi T, Mori H, Tomiyama T: Passive immunotherapy of tauopathy targeting pSer413tau: a pilot study in mice. Ann Clin Trans1 Neurol 2015, 2:241-255

11. Collin L, Bohrmann B, Gopfert U, Oroszlan-Szovik K, Ozmen L, Gruninger F: Neuronal uptake of tau/pS422 antibody and reduced progression of tau pathology in a mouse model of Alzheimer's disease. Brain 2014, 137:2834-2846

12. Chai X, Wu S, Murray TK, Kinley R, Cella CV, Sims H, Buckner N, Hanmer J, Davies P, O’Neill MJ, Hutton ML, Citron M: Passive immunization with anti-Tau antibodies in two transgenic models: reduction of Tau pathology and delay of disease progression. J Biol Chem 2011, 286:34457-34467

13. d'Abramo C, Acker CM, Jimenez H, Davies P: Passive immunization in JNPL3 transgenic mice using an array of phospho-tau specific antibodies. PLoS One 2015, 10:e0135774

14. Sankaranarayanan S, Barten DM, Vana L, Devidze N, Yang L, Cadelina G, Hoque N, DeCarr L, Keenan S, Lin A, Cao Y, Snyder B, Zhang B, Nitla M, Hirschfeld G, Barrezueta N, Polson C, Wes P, Rangan VS, Cacace A, Albright CF, Meredith J Jr, Trojanowski JQ, Lee VM, Brunden KR, Ahlijanian M: Passive immunization with phospho-tau antibodies reduces tau pathology and functional deficits in two distinct mouse tauopathy models. PLoS One 2015, 10:e0125614

15. Boutajangout A, Ingadottir J, Davies P, Sigurdsson EM: Passive immunization targeting pathological phospho-tau protein in a mouse model reduces functional decline and clears tau aggregates from the brain. J Neurochem 2011, 118:658-667

16. Castillo-Carranza DL, Sengupta U, Guerrero-Munoz MJ, LasagnaReeves CA, Gerson JE, Singh G, Estes DM, Barrett AD, Dineley KT, Jackson GR, Kayed R: Passive immunization with Tau oligomer monoclonal antibody reverses tauopathy phenotypes without affecting hyperphosphorylated neurofibrillary tangles. J Neurosci 2014, 34:4260-4272

17. Song L, Lu SX, Ouyang X, Melchor J, Lee J, Terracina G, Wang X, Hyde L, Hess JF, Parker EM, Zhang L: Analysis of tau posttranslational modifications in $\mathrm{rTg} 4510$ mice, a model of tau pathology. Mol Neurodegener 2015, 10:14

18. Barthelemy N, Fenaille F, Hirtz C, Sergeant N, Schraen-Maschke S, Vialaret J, Buee L, Gabelle A, Junot C, Lehmann S, Becher F: Tau protein quantification in human cerebrospinal fluid by targeted mass spectrometry at high sequence coverage provides insights into its primary structure heterogeneity. J Proteome Res 2016, 15:667-676

19. Barten DM, Cadelina GW, Hoque N, DeCarr LB, Guss VL, Yang L, Sankaranarayanan S, Wes PD, Flynn ME, Meredith JE, Ahlijanian MK, Albright CF: Tau transgenic mice as models for cerebrospinal fluid tau biomarkers. J Alzheimers Dis 2011, 24 Suppl 2:127-141

20. Martin L, Latypova X, Terro F: Post-translational modifications of tau protein: implications for Alzheimer's disease. Neurochem Int 2011, 58 : $458-471$

21. Santacruz K, Lewis J, Spires T, Paulson J, Kotilinek L, Ingelsson M, Guimaraes A, DeTure M, Ramsden M, McGowan E, Forster C, Yue M, Orne J, Janus C, Mariash A, Kuskowski M, Hyman B, Hutton M, Ashe KH: Tau suppression in a neurodegenerative mouse model improves memory function. Science 2005, 309:476-481

22. Hyman BT, Phelps CH, Beach TG, Bigio EH, Cairns NJ, Carrillo MC, Dickson DW, Duyckaerts C, Frosch MP, Masliah E, Mirra SS, 
Nelson PT, Schneider JA, Thal DR, Thies B, Trojanowski JQ, Vinters HV, Montine TJ: National Institute on Aging-Alzheimer's Association guidelines for the neuropathologic assessment of Alzheimer's disease. Alzheimers Dement 2012, 8:1-13

23. Goedert M, Spillantini MG, Cairns NJ, Crowther RA: Tau proteins of Alzheimer paired helical filaments: abnormal phosphorylation of all six brain isoforms. Neuron 1992, 8:159-168

24. Holmes BB, Furman JL, Mahan TE, Yamasaki TR, Mirbaha H, Eades WC, Belaygorod L, Cairns NJ, Holtzman DM, Diamond MI: Proteopathic tau seeding predicts tauopathy in vivo. Proc Natl Acad Sci U S A 2014, 111:E4376-E4385

25. Luo W, Liu W, Hu X, Hanna M, Caravaca A, Paul SM: Microglial internalization and degradation of pathological tau is enhanced by an anti-tau monoclonal antibody. Sci Rep 2015, 5:11161

26. Meredith JE Jr, Sankaranarayanan S, Guss V, Lanzetti AJ, Berisha F, Neely RJ, Slemmon JR, Portelius E, Zetterberg H, Blennow K, Soares H, Ahlijanian M, Albright CF: Characterization of novel CSF Tau and ptau biomarkers for Alzheimer's disease. PLoS One 2013, 8: e76523

27. Pooler AM, Phillips EC, Lau DH, Noble W, Hanger DP: Physiological release of endogenous tau is stimulated by neuronal activity. EMBO Rep 2013, 14:389-394

28. Yamada K, Cirrito JR, Stewart FR, Jiang H, Finn MB, Holmes BB, Binder LI, Mandelkow EM, Diamond MI, Lee VM, Holtzman DM: In vivo microdialysis reveals age-dependent decrease of brain interstitial fluid tau levels in P301S human tau transgenic mice. J Neurosci 2011, 31:13110-13117

29. Funk KE, Mirbaha H, Jiang H, Holtzman DM, Diamond MI: Distinct therapeutic mechanisms of tau antibodies: promoting microglial clearance versus blocking neuronal uptake. J Biol Chem 2015, 290: 21652-21662

30. Congdon EE, Gu J, Sait HB, Sigurdsson EM: Antibody uptake into neurons occurs primarily via clathrin-dependent Fcgamma receptor endocytosis and is a prerequisite for acute tau protein clearance. J Biol Chem 2013, 288:35452-35465

31. Krishnamurthy PK, Deng Y, Sigurdsson EM: Mechanistic studies of antibody-mediated clearance of tau aggregates using an ex vivo brain slice model. Front Psychiatry 2011, 2:59

32. Bi M, Ittner A, Ke YD, Gotz J, Ittner LM: Tau-targeted immunization impedes progression of neurofibrillary histopathology in aged P301L tau transgenic mice. PLoS One 2011, 6:e26860

33. Boimel M, Grigoriadis N, Lourbopoulos A, Haber E, Abramsky O, Rosenmann H: Efficacy and safety of immunization with phosphorylated tau against neurofibrillary tangles in mice. Exp Neurol 2010, 224:472-485

34. Ittner A, Bertz J, Suh LS, Stevens CH, Gotz J, Ittner LM: Tau-targeting passive immunization modulates aspects of pathology in tau transgenic mice. J Neurochem 2015, 132:135-145

35. Serrano-Pozo A, Frosch MP, Masliah E, Hyman BT: Neuropathological alterations in Alzheimer disease. Cold Spring Harb Perspect Med 2011, 1:a006189 